\title{
A new mechanism for dislocation blocking in strained layer epitaxial growth
}

\author{
E. A. Stach, ${ }^{1}$ K.W. Schwarz, ${ }^{2}$ R. Hull, ${ }^{3}$ F. M. Ross, ${ }^{2}$ and R.M. Tromp ${ }^{2}$ \\ ${ }^{I}$ National Center for Electron Microscopy, LBNL, Berkeley, California 94720; \\ ${ }^{2}$ IBM Research Division, T. J. Watson Research Center, P.O. Box 218, Yorktown Heights, New York 10598; \\ ${ }^{3}$ Department of Materials Science and Engineering, University of Virginia, Charlottesville, Virginia 22903;
}

\begin{abstract}
Dislocation interactions play a critical role in plasticity and heteroepitaxial strain relaxation. We use real time transmission electron microscopy observations of the interaction between threading and misfit dislocations in SiGe heterostructures to investigate interactions quantitatively. In addition to the expected long range blocking of threading segments, we observe a new short range mechanism which is significantly more effective. Simulations show that this reactive blocking occurs when two dislocations with the same Burgers vector reconnect.
\end{abstract}


The stability of semiconductor heterostructures during both growth and annealing is determined by the kinetics of misfit dislocation nucleation, propagation, and interaction. ${ }^{1}$ Dislocation interactions in particular not only alter the rate at which dislocations propagate, but also can halt threading dislocation motion entirely. Immobile threading segments are particularly deleterious to the performance of electronic devices because they provide a large number of traps at which electrical carriers can recombine, ${ }^{2}$ and they may act as pathways for the diffusion of dopants or impurities. Additionally, blocked dislocations can alter surface morphology as well as limit the overall relaxation of technologically important lowdislocation-density, graded buffer structures. ${ }^{3}$ To date there has been a number of analytical $^{4-8}$ and numerical ${ }^{9-12}$ descriptions of the dislocation - dislocation interaction process in heteroepitaxial films, but a careful experimental investigation of the processes has not been reported.

In this Letter, we use real time in-situ transmission electron microscopy to study the behavior of interacting dislocations during growth and annealing of SiGe / Si (001) heterostructures. These experiments allow us to determine the regime of epilayer thickness and composition where dislocation interactions halt the motion of a threading segment. We find that dislocation motion can be impeded by these interactions at higher levels of epilayer strain than is predicted by the most recent theoretical models. This is due to a particular reaction that occurs when the dislocations have parallel Burgers vectors. A numerical simulation of the interaction between dislocations is presented, and it is found that the observed blocking behavior is due to a reconnection reaction that occurs between these dislocations. This reactive blocking mechanism is an entirely different phenomenon from 
that discussed in prior experimental and theoretical studies of dislocation interactions in heteroepitaxial systems.

As a propagating threading dislocation approaches an interfacial misfit dislocation segment, the strain fields associated with each dislocation begin to overlap, resulting in an interaction force. The analytical solution of the magnitude of these interaction forces is quite complex, ${ }^{6,8}$ but has the general form: ${ }^{13}$

$$
\sigma_{\text {int }} \propto \frac{\mathbf{b}_{1} \cdot \mathbf{b}_{2}}{r}
$$

where $\mathbf{b}_{1}$ and $\mathbf{b}_{2}$ are the Burgers vectors of the two dislocations and $r$ is the distance between them. Thus, this force can act to either increase or decrease the magnitude of the net stress that drives the threading dislocation forward, depending on the signs of the Burgers vectors of the dislocations involved and the distance between them.

The net stress acting on a given segment of dislocation line, $\partial l$, is changed by this stress as follows: ${ }^{14}$

$$
\delta \sigma_{e x}=\delta \sigma_{a}-\delta \sigma_{T}-\delta \sigma_{\text {int }}(l) \quad \text { Equation } 2
$$

where $\delta \sigma_{e x}$ is the net excess stress in the epilayer, $\delta \sigma_{a}$ is the stress caused by the epitaxial misfit, $\delta \sigma_{T}$ is the back stress arising from the line tension of the dislocation segment and $\delta \sigma_{\text {int }}(l)$ is the interaction back stress. Thus, if $\delta \sigma_{\text {int }}(l) \geq \delta \sigma_{a}-\delta \sigma_{T}$ on a particular segment of the threading dislocation, the net force on that segment is less than or equal to zero. If this interaction stress cancels the other stresses over a significant portion of the threading segment, the motion of the entire dislocation will be halted. This is what is conventionally termed dislocation blocking. As we will show below, this long-range blocking is but one type of interaction observed in these layers. If the interaction stress is not sufficiently large, the threading dislocation will propagate past the interfacial segment. 
To date, the analytical ${ }^{6,8}$ and numerical ${ }^{9-12}$ models of dislocation interactions in heteroepitaxial films have focused on determining the epilayer thickness and composition at which the conventional long-range blocking condition is met. In the models of Freund ${ }^{6}$ and Gosling, et al. ${ }^{8}$ isotropic elasticity theory is used to determine the magnitude of the interaction force between the two perfect dislocations, and this force is incorporated into the net driving stress on the dislocation following the framework of Equation 2. Because the analytical models assume a fixed shape for the threading arm, they significantly overestimate the strength of the blocking effect. Schwarz ${ }^{9-11}$ as well as and Kukta and Freund ${ }^{12}$ have therefore used numerical approaches to investigate the dynamical interactions between perfect dislocations in these systems. Schwarz applied the full three-dimensional PeachKoehler equation to interactions in $\mathrm{Si} / \mathrm{SiGe} / \mathrm{Si}(001)$ structures and found dislocation blocking to be a relatively minor effect in capped layers - only occurring when the epilayer strain is less than $20 \%$ greater than the Matthews - Blakeslee critical strain. ${ }^{15}$ Kukta and Freund's numerical simulations for dislocation interactions in uncapped layers found dislocation blocking to occur up to strains of $35-50 \%$ above the critical strain. The difference between these results may lie in the different treatment of surface effects in each. However, a complete treatment of the interaction between a threading dislocation and a free surface has not been attempted, so that the issue of exactly how large these effects are is not yet fully resolved. As we report in this paper, however, a completely different blocking mechanism exists which appears to be more significant than the long-range effects considered previously.

In order to gain experimental insight into the mechanics of dislocation interactions in these systems, we observed interactions in real time during both growth and annealing using 
an ultrahigh vacuum transmission electron microscope (UHV-TEM) equipped with in-situ chemical vapor deposition (CVD) capabilities. This microscope is a modified $300 \mathrm{kV}$ Hitachi H9000 TEM with a base pressure of $2 \times 10^{-10}$ Torr in the specimen region. A simple gas handling system permits the introduction of disilane and digermane onto a heated specimen, allowing direct observation of the CVD SiGe growth process in real time at high spatial resolution. Details of the experimental methods have been given in a previous publication. ${ }^{16}$ Additional observations of dislocation interactions were made during postgrowth annealing of metastable structures grown by molecular beam epitaxy (MBE). These experiments were performed using a high angle double-tilt heating goniometer in a JEOL 2000FX transmission electron microscope (TEM). Tilting experiments allow determination of dislocation Burgers vectors through interpretation of changes in diffraction contrast.

The unique in-situ growth capability of the UHV-TEM permits efficient exploration of the behavior of dislocation interactions as a function of epilayer thickness and composition. In these experiments, SiGe epilayers are grown until they exceed the critical thickness for misfit dislocation formation. ${ }^{15}$ Due to the relatively slow relaxation kinetics in SiGe a metastable strain remained in the film at this point. ${ }^{1}$ Film growth was halted, and the sample was annealed thereafter in order to observe dislocation interactions directly. Dislocation motion was observed using two-beam 220 bright-field or dark-field images, and the resulting images were recorded onto videotape via a slow-scan CCD camera at sampling rates of 15 or 30 frames per second. By monitoring the initiation of threading dislocation motion, it is possible in the UHV-TEM to halt crystal growth while the metastable excess stress in the film is still relatively low. This is important, as dislocation blocking is predicted to be most significant during growth and annealing in this low stress regime. ${ }^{4-12}$ Following 
observations of dislocation interactions during this annealing period, epilayer growth was begun again in order to observe dislocation interactions at higher epilayer thicknesses within the same film.

In Figure 1 we present the data from a number of UHV-TEM deposition experiments. Shown are the epilayer thicknesses and strains (which are directly proportional to composition via $\varepsilon \approx-0.042 x)$ at which different types of interactions were observed. In general, we find three different regimes of interaction behavior. In one growth run the epilayer thickness and germanium concentration were both relatively low - i.e. the net excess stress driving dislocation motion was low - and every interaction between dislocations resulted in blocking of the threading segment. Thus, in this sample the long-range blocking mechanism described above was dominant. It was observed in this sample that dislocation threading segments became blocked both before reaching the interfacial segment, as well as when the bottom of the threading segment and interfacial segment meet at the heteroepitaxial interface.

In the other deposition runs, we saw a surprisingly different behavior. In these cases, certain specific interactions were observed for which the blocking was particularly strong, occurring at epilayer thicknesses and compositions far in excess of the Matthews-Blakeslee critical thickness. At these same thicknesses, other interactions did not result in blocking at all. Figure 2 presents a post-mortem TEM image showing a number dislocation intersection events. It can be seen that when dislocation intersections occur a variety of interactions can take place, each having its own distinctive geometrical signature. Of particular interest here are the events marked by stars in Figure 2, where the motion of the threading arms has been arrested. Burgers vector determination of the dislocations that showed this particular 
reaction were performed ex-situ in a JEOL 2000FX using $(\mathbf{g} \cdot \mathbf{b}) s$ analysis. ${ }^{17}$ This analysis established that this type of interaction occurs only when the two dislocations have parallel Burgers vectors. The dislocation interactions that are arrowed in the figure also had parallel Burgers vectors, but no blocking was observed.

The source of the strong blocking effect seen in Figure 2 can be elucidated by numerical simulations. These were done using the PARANOID dislocation dynamics code, the basic features of which are described in Reference 11. In our simulations, a threading dislocation is allowed to propagate towards a misfit dislocation on an intersecting glide plane, and the resulting behavior is observed. With the available Burgers vectors and directions of approach, sixteen distinct interactions of this kind are possible, leading to a variety of outcomes such as repulsion of the misfit dislocation into the substrate, reconnection of the two dislocations, and junction or jog creation. We have calculated all 16 of these, but for the present paper content ourselves with pointing out that four of the interactions involve parallel Burgers vectors and can result in a reconnection reaction. Of these, two $\operatorname{cases}^{18}$ are of particular interest because they result in the situation illustrated in Figure 3.

The evolution of the dislocation interaction in Figure $3 \mathrm{a}$ is as follows. A threading arm coming in from the left on glide plane 1 (red) reconnects to the intersecting misfit dislocation on glide plane 2 (blue), forming two dislocations having a segment on each glide plane. Because the intersection edge formed by the two glide planes is not normal to the layer, line tension causes edge point A to ride up the glide-plane edge (green), while edge point B moves downward into the substrate. For the two particular cases of interest here, this results in that part of the threading segment that lies between A and the surface becoming 
shorter as shown in the figure. To move such a segment requires a higher strain, and in fact, the situation shown here is a stationary state. By gradually increasing the layer strain, one can determine the strain at which this configuration becomes unstable, and the threading arm again begins to propagate.

We have calculated the maximum blocking strain for this mechanism as a function of layer thickness. The two cases of interest are not equivalent, and give slightly different results. The upper curve in Figure 1 shows the calculated values averaged between the two cases. We also show the Matthews - Blakeslee critical thickness, calculated with the identical computational settings. The calculations are subject to the same reservations concerning absolute accuracy that we have expressed about earlier results. Nevertheless, we see from Figure 1 that there is good agreement with experiment. In addition, Figure $3 \mathrm{~b}$ shows that the experimentally observed geometrical signature of the reconnection-blocked state is predicted by the simulation with remarkable accuracy. Finally, we point out that both theory and experiment show this phenomenon only when the Burgers vectors of the two dislocations are parallel. It seems clear, therefore that the observed strong blocking mechanism is a straightforward geometrical consequence of a reconnection between the threading and misfit dislocations. The geometrical signature makes it clear that this is a new mechanism, having nothing to do with the long-range blocking mechanism treated in References 6 through 12.

The remaining two possible interactions for which the Burgers vectors are parallel correspond to allowing the threading dislocations to approach the misfit line from the opposite side. Reconnection is still observed to occur in the simulations, but for these cases the reconnection reaction is not observed to lead to blocking. This is because the edge point 
of the threading segment is not forced towards the surface in the same manner as shown in Figure 3. As indicated by the arrows in Figure 2, several events of this type are also observed experimentally. Again, the experimental and numerical simulations show remarkable correspondence.

In this Letter, we have delineated the range of epilayer thicknesses and compositions at which the motion of epilayer threading dislocations may be halted by interaction with preexisting interfacial segments during heteroepitaxial growth and annealing. We find that these interactions can halt dislocation motion at levels of strain that are substantially above the critical thickness. At the highest levels of strain those dislocations that become blocked are those that have parallel Burgers vectors. Numerical simulations show that for these cases, the dislocations undergo a reconnection at the interface that causes the blocking. This is in contrast to the mechanism of blocking predicted by prior theoretical discussion, which postulate that dislocation blocking is solely a result of long range elastic repulsion between dislocations. Our results indicate that dislocation blocking can limit the rate of heteroepitaxial strain relaxation at epilayers thicknesses in excess of present models.

Acknowledgments: This work was supported by the NSF via DMR \# 9531696 and by IBM as a Joint Study Program. The authors would like to thank M. C. Reuter, L. B. Freund, J. C. Bean and W. A. Jesser for helpful comments and assistance. One of the authors (E. A. S.) would also like to thank IBM for support through a Cooperative Fellowship. 


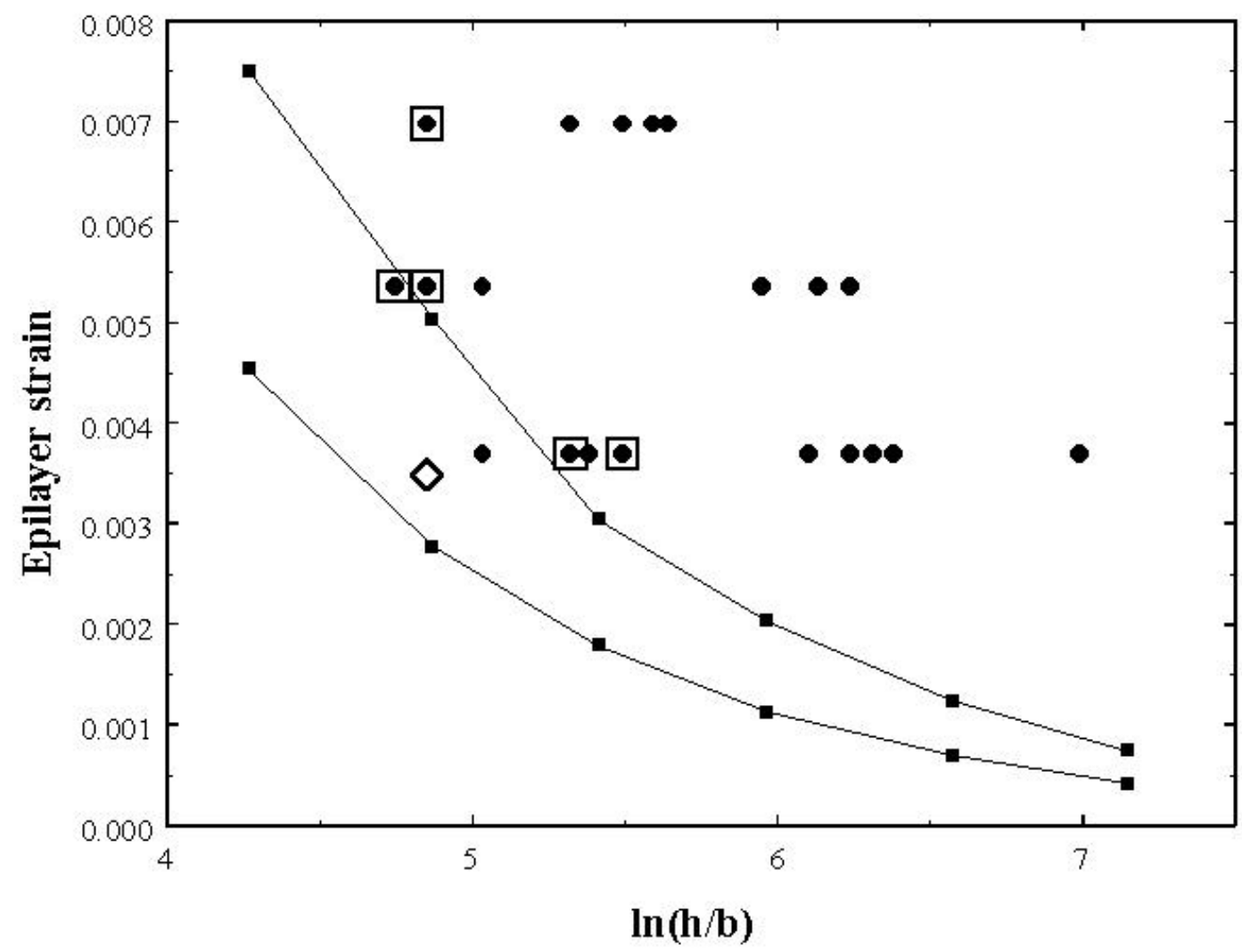

Figure 1. Epilayer thickness and strain at which dislocation interactions were observed. Hollow diamond - long-range blocking interactions observed; solid circles - nonblocking interactions observed; hollow squares - reactive blocking interactions observed for parallel Burgers vectors, other combinations of Burgers vectors are non-blocking (also shown). The upper curve shows the limits of reactive blocking calculated by the simulation program; the lower curve shows the critical thickness computed by the same methods. 


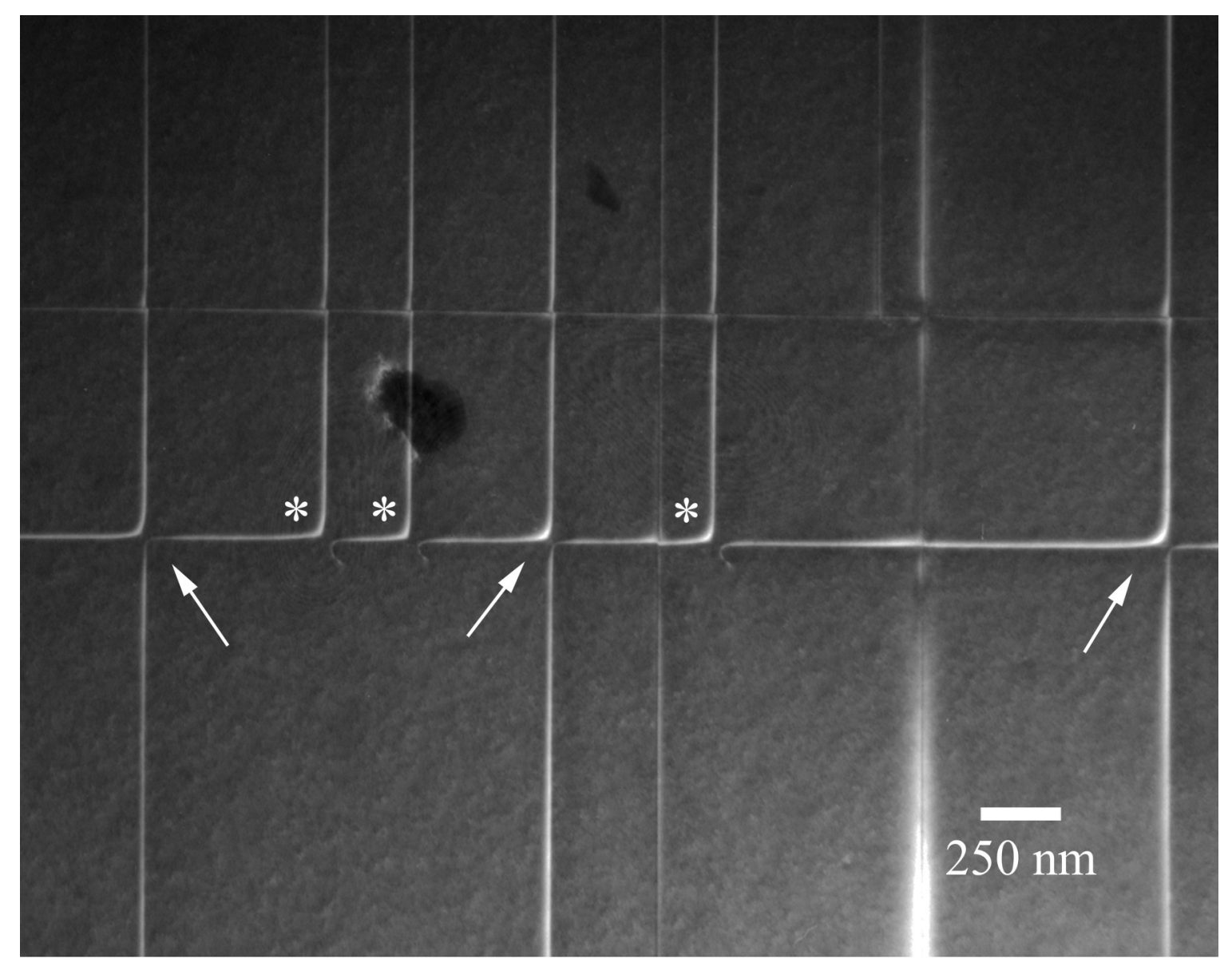

Figure 2. Dark field $\mathbf{g}=400 s<<0$ image showing dislocation interactions in a $70 \mathrm{~nm}$ $\mathrm{Si}_{80} \mathrm{Ge}_{20} / \mathrm{Si}(001)$ heterostructure. Dislocation pairs with parallel Burgers vectors are indicated by a $(*)$ if the reaction results in blocking and are arrowed in the cases where the interaction does not result in blocking. 

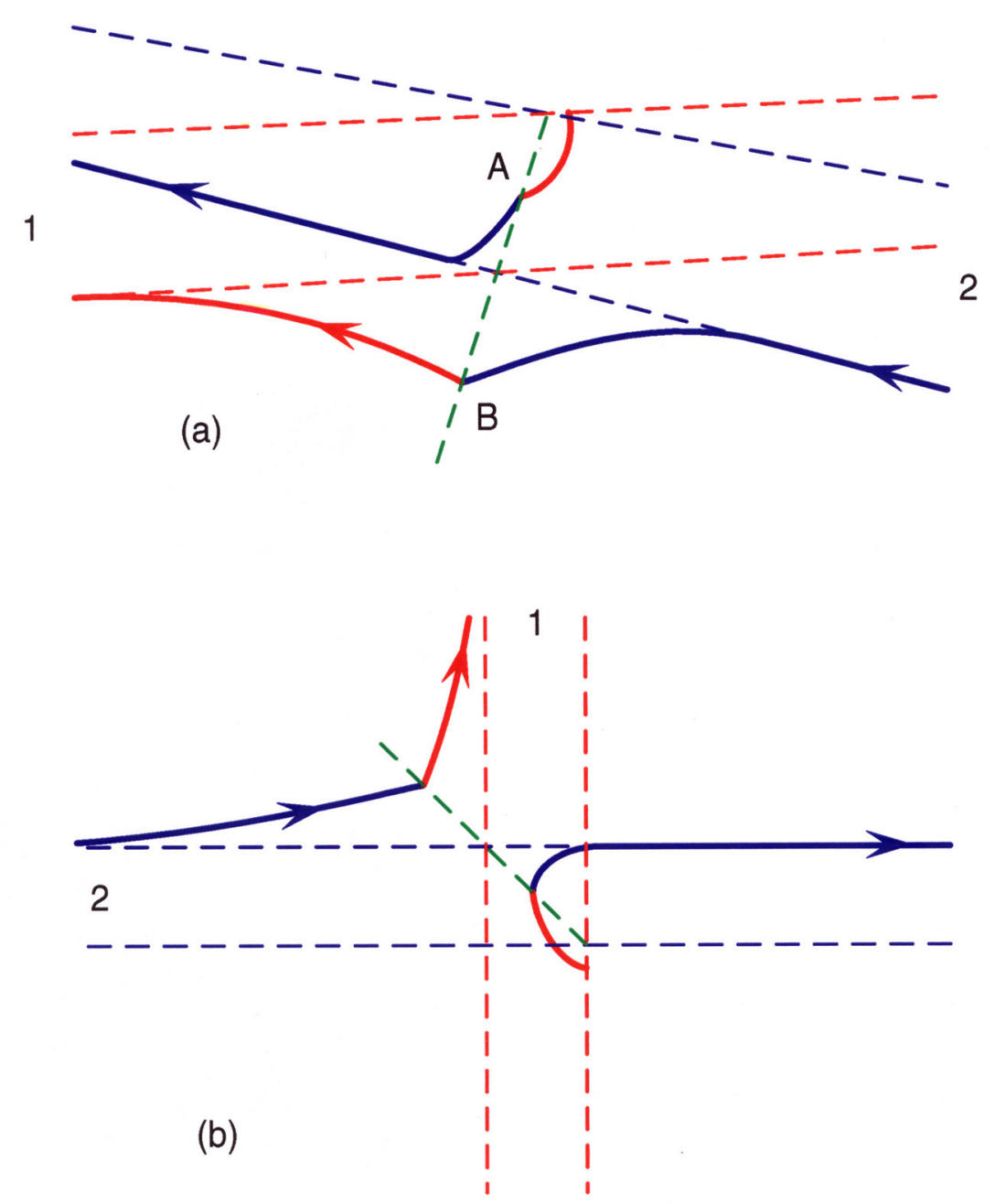

Figure 3. (a) Stationary configuration formed by the interaction of a threading dislocation on the (111) glide plane with a misfit dislocation on the (111) glide plane. The Burgers vector of both segments is $1 / 2\left[\begin{array}{lll}0 & 1 & 1\end{array}\right]$. The layer thickness here is $50 \mathrm{~nm}$ and the strain is 0.0048 . (b) Plan view of the same configuration, rotated to match the perspective of Figure 2. Those parts of the dislocations lying on the $(\overline{1} 11)$ glide plane are shown in red, those on the (111) glide plane in blue. 


\section{References}

${ }^{1}$ For a recent review of strain relaxation in $\mathrm{SiGe} / \mathrm{Si}$ heterostructures see R. Hull and E.A. Stach, "Strain accommodation and relief in SiGe / Si heteroepitaxy" in Heteroepitaxy, Thin Film Systems, A.W.K. Liu and M.B. Santos (ed.). Rivers Edge, NJ: World Scientific Publishing Co., Inc. (1999) in press.

${ }^{2}$ F.M. Ross, R. Hull, D. Bahnck, J.C. Bean, L.J. Peticolas and C.A. King, Appl. Phys. Lett. 62, 1426 (1993).

${ }^{3}$ S.R. Samavedam and E.A. Fitzgerald, J. Appl. Phys. 81, 3108 (1997).

${ }^{4}$ B.W. Dodson, Appl. Phys. Lett. 53, 37 (1988).

${ }^{5}$ R. Hull and J.C. Bean, Appl. Phys. Lett. 54, 925 (1989); R. Hull, J.C. Bean and C. Buescher, J. Appl. Phys. 665837 (1989).

${ }^{6}$ L.B. Freund, J. Appl. Phys. 68, 2073 (1990); J. Mech. Phys. Solids, 38, 657, (1990).

${ }^{7}$ E.P. Kvam, Phil. Mag. Lett. 62, 167 (1990).

${ }^{8}$ T.J. Gosling, S.C. Jain and A.H. Harker, phys. stat. sol(a) 146, 713 (1994).

${ }^{9}$ K.W. Schwarz and J. Tersoff, Appl. Phys. Lett. 69, 1220 (1996).

${ }^{10}$ K.W. Schwarz, Phys. Rev. Lett. 78, 4785 (1997).

${ }^{11}$ K.W. Schwarz, J. Appl. Phys. 85, 108 (1999); J. Appl. Phys. 85120 (1999).

${ }^{12}$ R.V. Kukta, Ph.D disseration, Brown University 1998. R.V. Kukta and L.B. Freund, unpublished work.

${ }^{13}$ J.P. Hirth and J. Lothe, Theory of Dislocations, $2^{\text {nd }}$ ed., John Wiley, New York (1982).

${ }^{14}$ B.W. Dodson and J.Y. Tsao, Appl. Phys. Lett. 51, 1325 (1987).

${ }^{15}$ J.W. Matthews and A.E. Blakeslee, J. Cryst. Growth 27, 118 (1974); 29, 373 (1975); 32, 265 (1976). 
${ }^{16}$ E.A. Stach, R. Hull, R.M. Tromp, M.C. Reuter, M. Copel, F.K. LeGoues and J.C. Bean, J. Appl. Phys. 83, 1931 (1998).

17 P.B. Hirsch, A. Howie, R. Nicholson, D.W. Pashley, and M.J. Whelan, Electron Microscopy of Thin Crystals, Krieger, Malabar, FL (1977). See pages $162-194$ and $263-$ 271.

${ }^{18}$ For compressive strain, with the misfit dislocation initially oriented in the $[1 \overline{1} 0]$ direction on the (111) plane, and the threading segment moving in the $[\overline{1} \overline{1} 0]$ direction, the two cases of interest are $(i)$ a threading segment with slip system $\left(\begin{array}{lll}1 & 11\end{array}\right) \frac{1}{2}\left[\begin{array}{lll}0 & \overline{1} & 1\end{array}\right]$ interacting with a (111) $\frac{1}{2}\left[\begin{array}{ll}0 & 1 \\ 1\end{array}\right]$ interfacial segment, and a $\left(\begin{array}{lll}1 & 1 & 1\end{array}\right) \frac{1}{2}\left[\begin{array}{ll}1 & 01\end{array}\right]$ threading dislocation interacting with a (111) $\frac{1}{2}[\overline{1} 01]$ interfacial segment. 